Originally published as:

Soprana, E., Panigada, M., Knauf, M., Radaelli, A., Vigevani, L., Palini, A., Villa, C., Malnati, M., Cassina, G., Kurth, R., Norley, S., Siccardi, A.G.

Joint production of prime/boost pairs of Fowlpox Virus and Modified Vaccinia Ankara recombinants carrying the same transgene

(2011) Journal of Virological Methods, 174 (1-2), pp. 22-28.

DOI: 10.1016/j.jviromet.2011.03.013

This is an author manuscript.

The definitive version is available at: http://www.sciencedirect.com/ 


\title{
Joint production of prime/boost pairs of Fowlpox Virus and Modified Vaccinia Ankara recombinants carrying the same transgene
}

\author{
Elisa Soprana ${ }^{a}$, Maddalena Panigada ${ }^{a, b}$, Mathias Knauf ${ }^{c}$, Antonia Radaelli $^{d}$, Luisa Vigevani $^{\mathrm{a}, \mathrm{b}}$, Alessio \\ Palini $^{a}$, Chiara Villa ${ }^{a}$, Mauro Malnati ${ }^{a}$, Giulia Cassina ${ }^{a}$, Reinhard Kurth ${ }^{c}$, Stephen Norley ${ }^{c}$, Antonio G. \\ Siccardi ${ }^{a, b}$, \\ a San Raffaele Scientific Institute, Milano, Italy \\ b Dipartimento di Biologia e Genetica per le Scienze Mediche, Università di Milano, Italy \\ ${ }^{c}$ Robert Koch Institut, Berlin, Germany \\ d Dipartimento di Scienze Farmacologiche e Istituto di Neuroscienze del CNR, Sezione di \\ Farmacologia Celluare e Molecolare, Università di Milano, Italy
}

Received 7 December 2010. Revised 3 March 2011. Accepted 9 March 2011. Available online 16 March 2011.

\begin{abstract}
Pairs of recombinant MVA (Modified Vaccinia Ankara) and FPV (Fowlpox Virus) expressing the same transgene are reasonable candidates for prime/boost regimens, because cross-reacting immune responses between the two vectors, both non-replicative in mammalian hosts, are very limited. The acceptor virus FPD-Red, a derivative of FPV, carrying a red fluorescent protein gene flanked by the homology regions of MVA deletion III, was constructed. The same MVA Transfer Plasmid Green, designed to insert transgenes into the MVA deletion III locus, can therefore be used to transfer transgenes into both acceptor viruses MVA-Red and FPD-Red with the described recently Red-toGreen gene swapping method. Cells infected by either recombinant virus can be sorted differentially by a simple and reliable FACS-based purification protocol. The procedure is carried out in primary chick embryo fibroblasts grown in serum-free media and was applied to the production of three rMVA/rFPV pairs expressing the H5N1 avian influenza antigens M1, M2 and NP. The viral genes were human codon-optimized and expressed at high levels in both chick and mammalian cells. Both singlestep and multiple-step growth analyses showed no significant differences in growth due to the transgenes in either rMVA or rFPV derivatives.
\end{abstract}

\section{Introduction}

Both MVA (Modified Vaccinia Ankara) and FPV (Fowlpox Virus) poxviral strains are considered suitable human vaccine vectors because of the high expression of heterologous genes and the lack of replication in mammalian cells ( [Sutter and Moss, 1992] and [Taylor et al., 1988]).

Fowlpox recombinants encoding tumor ( [Grosenbach et al., 2001], [Restifo et al., 1994] and [Wang et al., 1995]), or viral antigens ( [Feng et al., 2010], [Kent et al., 1998], [Kent et al., 2000], [Radaelli et al., 2007] and [Steensels et al., 2009]) have been shown to elicit CD8 T cell responses in animal models.

MVA recombinants also proved to protect against several infectious diseases and tumors in various animal models ( [Barouch et al., 2001], [Carson et al., 2009], [Hirsch et al., 1996], [Schneider et al., 1998], [Sutter et al., 1994], [Weidinger et al., 2001], [Wyatt et al., 1996], [Carroll et al., 1997] and [Drexler et al., 1998]) and have been used widely in prime-boost strategies (Amara et al., 2002) and in clinical trials ( [Cosma et al., 2003], [Jaoko et al., 2008], [Marshall et al., 2005], [McConkey et al., 2003] and [Webster et al., 2006]). 
Despite their relatedness, cross-reacting immune responses between the two vectors are very limited ( [Hodge et al., 2003] and [Tsang et al., 2005]). These features make MVA and FPV ideal vectors for both prophylactic and therapeutic human vaccines using a prime/boost regimen to stimulate both the cellular and humoral arms of the immune system with high efficiency.

A quick and reliable method to produce marker-free rMVA by swapping green and red fluorescence genes combined with fluorescence-activated cell sorting has been described previously (Di Lullo et al., 2010). To facilitate the production of pairs of recombinant MVA and FPV expressing the same transgene, the method was extended to the production of rFPVs. To achieve this, an acceptor virus FPD-Red, a derivative of FPV containing an active red fluorescent protein gene flanked by the homology regions of MVA deletion III within the fp9.046 gene, was constructed. Consequently, the same Transfer Plasmid Green, designed to insert transgenes into the MVA deletion III borders by homology recombination (Di Lullo et al., 2010) can be used to transfer transgenes into both MVA-Red and FPD-Red acceptor viruses.

\section{Materials and methods}

\subsection{Construction of recombinant virus FPD-Red}

The plasmid plll-sP-Red (Di Lullo et al., 2010) was digested with Spel, blunted by Klenow fragment and digested again with EcoRI to obtain a 1808-bp fragment containing the HcRed1.1 gene (under the synthetic VV promoter SP) flanked by the MVA-deletion-III homology regions. The fragment was inserted into the EcoRV and EcoRI sites of pBlueScript SK(-) to obtain the pSK(-)Red intermediate, from which the HindlII-Smal 1836 bp fragment was derived and inserted into the HindIII and Nrul sites within the FPV fp9.046 gene 3- $\beta$-hydroxysteroid dehydrogenase 5-delta 4 isomerase, using the recombinant pFP128 plasmid after excision of the gene inserted previously ( [Pozzi et al., 2009] and [Radaelli et al., 2007]). The resulting pFP-Dual-Red Tranfer Plasmid was transfected $(10 \mu \mathrm{g})$ into chick embryo fibroblasts (CEF) prepared and grown in serum-free medium (VP-SFM; GIBCO) and infected with FPV wild-type (FPwt) (0.05 m.o.i.) ( [Pozzi et al., 2009] and [Radaelli et al., 2007]). The lysate derived from such infection/transfection was diluted $1 / 50$ and used to infect CEF in the presence of $1 \mathrm{mM}$ cytochalasin D (Di Lullo et al., 2010). Single-red cells were sorted onto CEF microcultures and the recombinant virus FPV-Dual-Red (FPD-Red) was cloned by two rounds of terminal dilution in microcultures. These cultures were monitored by whole-plate Typhoon fluoroimaging, which allows green or red fluorescence in microcultures to be visualized (Molecular Dynamics Typhoon 8600 Imager).

\subsection{Construction of plasmids carrying H5N1 M1, M2 and NP}

Wild-type and human codon-optimized (co) co-M1, co-M2 and co-NP genes from H5N1 Influenza A/Vietnam/1194/2004 were synthesized (Kotsopoulou et al., 2000) and PCR-elongated after inserting HindIII and BamHI restriction sites and a C-terminal sequence containing the coding sequence for the 9 amino acid V5-tag (MDDLGSIPNPLLGLD) (Southern et al., 1991). Codon-optimized sequences are reported in Table S1 of Supplementary Materials. Wild-type and modified genes were cloned into the pTH plasmid (Hanke et al., 1998) to yield plasmids pTH-wtM1, pTH-wtM2, pTH-wtNP, pTH-coM1, pTH-coM2, pTH-coNP. The human codon-optimized genes were then PCR-elongated after inserting $\mathrm{BamHI}$ and Ascl restriction sites and subcloned into the BamHI-Ascl sites of Transfer Plasmid Green (TPG) (Di Lullo et al., 2010) to yield plasmids TPG-coM1, TPG-coM2 and TPG-coNP.

\subsection{Production of MVA and FPD recombinants}

Primary CEF were infected/transfected with MVA-Red or FPD-Red at an m.o.i. of 0.05 and TPGs carrying either co-M1, coM2 or co-NP transgenes (as well as the EGFP marker gene). The virus lysates, derived from infection/transfection and diluted 1:100, were used to infect fresh CEF in the presence of $1 \mu \mathrm{M}$ cytochalasin D (Sigma-Aldrich, St. Louis, MO). Infected cells, collected $24 \mathrm{~h}$ p.i. by trypsinization, were washed and kept on ice. Green cells were either bulk- or single cell-sorted by a Becton Dickinson FACS Vantage SE flow cytometer (Becton Dickinson, San José, CA). EGFP fluorescence (excited at $488 \mathrm{~nm}$ ) was detected using a 530/30 nm bandpass filter. HcRed1-1 
fluorescence (excited at $633 \mathrm{~nm}$ ) was detected using a 660/20 nm bandpass filter. Sorted cells were seeded onto CEF monolayers in microplate cultures to produce virus lysates. Finally, markerless recombinant viruses (rMVA-M1, rMVA-M2, rMVA-NP, rFPD-M1, rFPD-M2, rFPD-NP) were cloned by terminal dilution and expanded in CEF by conventional methods.

\subsection{Virus titration}

Terminal dilution titration and cloning of rMVA and rFPD were carried out in 96-well plates, by infecting 8 or 16 CEF microcultures with each serial 10 -fold dilution of viral lysates. In order to spread viral infection throughout the monolayers, CEF microcultures were "scrambled" after $48 \mathrm{~h}$ by scraping the monolayers and pipetting up and down with the multichannel pipette. After an additional $48 \mathrm{~h}$, the microcultures were monitored by fluorescence microscopy or by whole-plate fluoroimaging (Typhoon, GE Healthcare) to distinguish uninfected from infected cultures. A macroscopic record of viral titrations could also be obtained by twice freezing and thawing the microtiter plates and replica-plating $50 \mu \mathrm{l}$ of the supernatants onto BHK-21 cells (seeded freshly at 2000 cells/well). For MVA-infected cultures the replica-plating was carried out after 4 days and for FPV-infected cultures after 6-7 days. Four days later, BHK-21 microcultures are fixed by acetone-methanol, washed with water and stained with crystal violet. MVA grows in BHK-21 and therefore only uninfected monolayers persist and are stained. Conversely, FPV does not grow in BHK-21 cells, but infection prevents their growth and the formation of a cell monolayer. Marker-free (non-fluorescent) segregants of rMVAs and rFPDs can be identified by comparing whole-plate fluorograms with crystal violet-stained replicas, selecting those cultures in which neither fluorescence nor staining occurs.

The mean number of infecting viruses per well $(m)$ was calculated using Poisson's distribution formula $(m=-\ln f)$ for the rows of microwells containing a fraction of uninfected cultures $(f)$.

\subsection{Transgene expression analysis}

Western Blot. CEF, BHK-21 or HEK-293-T cells (ca. $1 \times 10^{6}$ ) infected with rMVA or rFPD at an m.o.i. 5 (or mock-infected) were lysed with $1 \%$ NP40 in PBS and centrifuged for 5 min at 10,000 $\times g$. The supernatants were incubated for $5 \mathrm{~min}$ in reducing sample buffer $(30 \mathrm{mM}$ Tris- $\mathrm{HCl}, \mathrm{pH} 6.8,1.5 \% \mathrm{SDS}$, $10 \mathrm{mM}$ beta-mercaptoethanol, $10 \%$ glycerol, $0.1 \mathrm{mg} / \mathrm{mL}$ bromophenol blue) at $100{ }^{\circ} \mathrm{C}$. Protein samples were then resolved by $12 \%$ SDS-PAGE and transferred to nitrocellulose. Avian influenza proteins were detected using chick anti-H5N1 polyclonal antibodies (Istituto Zooprofilattico Sperimentale Tre Venezie, Padova, Italy), monoclonal antibodies SC-66143, SC-66143, SC-66143 (Santa Cruz, Heidelberg, Germany), or anti-V5 monoclonal antibody V5-10 (Sigma-Aldrich) followed by HRP-conjugated rabbit anti-chicken Ig or anti-mouse Ig antibodies (Jackson Immunoresearch Laboratories Inc., West Grove, PA, USA). Western blots were developed by enhanced chemiluminescence (ECL; Amersham Biosciences, Milan, Italy) autoradiography.

Foci immunostaining. Immunoperoxidase assays to detect rMVA- or rFPD-infected foci were performed on paraformaldehyde-fixed monolayers of CEF employing the same primary and secondary antibodies as above and the aminoethylcarbazole chromogenic substrate (Sigma-Aldrich).

\subsection{Growth parameters of recombinant viruses}

Burst size (average number of viruses produced by cells infected at 10 m.o.i.) and growth rate were measured by single-step and multiple-step growth analysis for all the recombinants and the corresponding acceptor viruses. The burst size was calculated by dividing the total number of viruses in CEF cultures at $48 \mathrm{~h}$ (for MVA derivatives), or $72 \mathrm{~h}$ (for FPV derivatives) by the number of cells present in culture at the time of infection. The growth rate was calculated as the doubling time of the virus titre measured on the exponential curve from 3 to 4 time points $(12,16,20,40 \mathrm{~h}$ for MVA; 19, 24, 28, $48 \mathrm{~h}$ for FPV) in CEF cultures infected at an m.o.i of 0.05 . 


\subsection{Real-time PCR}

A Real-time PCR assay for FPV DNA quantitation was based on the conserved region of the fp9,046 gene carried by plasmid pFPwt. The forward and reverse primers, which amplify a 73-bp fragment and a probe of $18 \mathrm{bp}$ complementary to an internal region $1 \mathrm{bp}$ downstream of the forward primer, were selected using the Primer Express software (Applied Biosystem, Foster City, CA, USA) (Table S2 of Supplementary Materials). The thermodynamic features were predicted by the Oligo 6 software (Molecular Biology Insights Co, USA). The primers were synthesized by Primm (Milan, Italy), whereas the 3'-minor groove binder DNA probe, synthesized with the reporter dye 6-carboxy-fluorescein linked covalently to the $5^{\prime}$ end of the sequence, was prepared by Applied Biosystems (Warrington, United Kingdom). An extensive search in the EMBL, BLAST and GenBank databases indicated that neither the primers nor the probe shared significant homology with other known nucleotide sequences. The reaction was optimized by determining the concentrations of primers and probes and the annealing temperature yielding the highest intensity of reporter fluorescent signal without a reduction in specificity or sensitivity. CEF were seeded in 48-well plates at 40,000 cells/culture. At 24 h, FPV derivatives were added at an m.o.i. of 0.1 . Two hours later the cells were washed and the complete medium restored. At various time points $(18,24,48$ and $64 \mathrm{~h})$ the monolayer was washed and incubated with a lysis reagent described previously (Malnati et al., 2008) and proteinase $\mathrm{K}$ for $2 \mathrm{~h}$ at $56{ }^{\circ} \mathrm{C}$, followed by a $15 \mathrm{~min}$ incubation at $95^{\circ} \mathrm{C}$. A fraction of the lysate was used in TaqMan Realtime PCR for FPV DNA quantitation. Details of the TaqMan reaction are reported in Table S2 (in Supplementary Materials). A standard curve was prepared with serial dilutions of known input target copies ( $x$ axis) vs. the corresponding $C t$ values ( $y$ axis) using the least-squares fit method (Fig. S2 in Supplementary Materials).

\section{Results}

\subsection{Production of an acceptor FPV suitable for gene swapping}

In order to extend to FPV the Red-to-Green gene swapping method developed recently for MVA ( [Di Lullo et al., 2009] and [Di Lullo et al., 2010]), and simplify the construction of rMVA/rFPV pairs for prime/boost vaccine regimens, the MVA deletion III flanking regions were inserted into FPV, rendering it suitable for homologous recombination with the same Transfer Plasmid Green used for the construction of rMVA. This was achieved by cloning the red fluorescent protein gene HcRed1.1 (flanked by MVA Deletion III flanking regions) within the FPV homology regions of plasmid pFP128 ( [Pozzi et al., 2009] and [Radaelli et al., 2007]).

The resulting pFP-Dual-Red (Fig. 1a) containing both sets of homology regions of FPV and MVA, could be used for homologous recombination. Following infection/transfection of serum-free CEF with the pFP-Dual-Red Transfer Plasmid and FPwt, the recombinant FP-Dual-Red (FPD-Red) virus was obtained by sorting red infected cells (Fig. 1b) and two rounds of cloning by terminal dilution (Fig. 1c). Foci of FPD-Red infected CEF are shown in Fig. 1d.

\subsection{Construction of recombinant MVA and FPD carrying H5N1 avian influenza genes}

The sequences of M1, M2 and NP genes of a H5N1 avian influenza virus isolate (Influenza A/Vietnam/1194/2004) were codon-optimized for maximal expression in mammalian cells. Wild-type and codon-optimized synthetic genes, containing the $\mathrm{V} 5$ tag at the $\mathrm{C}^{\prime}$ terminus, were produced and cloned under control of the CMV promoter into the expression plasmid pTH, which is suitable for DNA vaccination. Expression of the wild-type (wt) and codon-optimized (co) genes in plasmid-transfected 293T cells was analysed by V5 Western blot analysis (Fig. 2a). The co form of the M1 gene was expressed at levels markedly higher than those of the wt form. An obvious increase in expression was also observed for the co form of the NP gene, while the M2 gene was expressed at similar levels for the wt and co forms.

The codon-optimized genes were also subcloned into Transfer Plasmid Green under the control of the P7.5 vaccinia promoter, and the correct gene expression was checked by V5 Western blot analysis of cell lysates following infection/transfection of CEF with plasmid DNA and MVA-Red (not shown). MVARed and FPD-Red (Fig. 2b) were then used as acceptor viruses for the construction of recombinants 
by the Red-to-Green gene swapping method. Following infection/transfection of CEF in serum-free medium, a cell lysate was prepared at $18 \mathrm{~h}$ p.i. and used to infect a fresh culture of CEF in the presence of cytochalasin $D$ to prevent superinfection by parental (red) virus of cells infected by the recombinant (green) viruses. Green infected cells were sorted in batch (Fig. 2c) and grown in CEF cells for 2 blind passages. Cloning by terminal dilution, followed by fluorography of the whole microtiter plate (Fig. 2d) allowed the isolation of green clones and a few marker-less clones for each of the transgenes, by comparing with a replica-plate of the microcultures stained by crystal violet.

\subsection{Characterization of transgene expression by recombinant viruses}

Transgene expression by recombinant MVA and FPD viruses was monitored by V5 immunofluorescence of infected CEF cells. Fig. 3a shows the expression of M1, M2 and NP avian flu antigens by FPD recombinant viruses. Three distinct morphological patterns for the three antigens were seen: M2 and NP displayed characteristic cytoplasmic membrane and nuclear localizations, respectively, while M1 displayed a granular/vesicular morphology. V5 Western blot analysis of all the six recombinants (Fig. 3b) confirmed the expected molecular weight of the recombinant proteins in both vector systems. The V5 immunoperoxidase morphology of infected foci in CEF monolayers is shown in Fig. 3c.

\subsection{Transgene insertion does not alter the growth parameters of recombinant viruses}

Wild-type, acceptor and recombinant viruses were analysed for their growth properties to verify whether the insertion/expression of the transgenes might impair virus replication. The burst size of all derivatives was determined by titrating the virus produced at $48 \mathrm{~h}$ (for MVA derivatives), or $72 \mathrm{~h}$ (for FPV/FPD derivatives) after infecting CEF at 10 m.o.i. Although the burst size of both MVA and FPV/FPD derivatives was unaffected by transgene insertion, it was significantly higher in MVA derivatives than in FPV/FPD (200 \pm 31 vs. $70 \pm 7$; average \pm se; $n=5 ; p=0.0017, t$ test).

The growth rate of MVA derivatives (Fig. 4a) was examined by titrating the virus produced at various time points after infecting CEF at 0.01 m.o.i. The exponential growth curves of all the derivatives could be described with a single equation (coefficient $=0.226 ; R^{2}=0.96$ ). The growth rate of FPV/FPD derivatives was analysed by virus titration and by real-time PCR. The two methods gave superimposable results (Fig. 4b). The exponential growth curves of all the derivatives, by both methods, could be described with a single equation (coefficient $=0.116 ; R^{2}=0.97$ ). The doubling time of FPV/FPD derivatives was significantly longer than that of MVA derivatives $(5.98 \mathrm{~h}$ vs. $3.48 \mathrm{~h}$; $p<0.0001$, likelihood ratio test).

\section{Discussion}

The Red-to-Green gene swapping method described recently for rMVA ( [Di Lullo et al., 2009] and [Di Lullo et al., 2010]) consists of a flow cytometry purification protocol. Single cells infected are sorted on the basis of differential fluorescence, which distinguishes the parental acceptor virus carrying a red gene from recombinants which have swapped the red gene with a cassette containing the transgene and a green marker gene from the transfer plasmid. In this way, a single cell type can be used throughout the whole procedure. Further novelties are the introduction of a reversible inhibitor of virus release (such as cytochalasin $\mathrm{D}$ ), which limits the infection to a single round, thus preventing superinfection by parental red viruses and the extension of the method to serum-free CEF, an essential step for its implementation in the field of human vaccine development.

In order to extend to FPV the Red-to-Green gene swapping method developed for MVA and simplify the construction of rMVA/rFPV pairs for prime/boost vaccine regimens, homology regions derived from MVA (Deletion III flanking regions) were introduced into a FPV derivative, making it suitable for homologous recombination with the same Transfer Plasmid Green used for the construction of rMVA (Di Lullo et al., 2010). This was achieved by cloning the red fluorescent protein gene HcRed1.1 (flanked by MVA Deletion III regions) within the FPV homology regions of plasmid pFPwt (Radaelli et al., 2007). 
The new method was used to construct 3 rMVA/rFPV pairs with M1, M2 and NP transgenes from H5N1 Influenza A/Vietnam/1194/2004, to be used in prime/boost vaccination regimens. The transgenes were codon-optimized to enhance expression in infected cells. This was verified experimentally by comparing the expression levels of wt and codon-optimized sequences in a human cell line (Fig. 2a). The increase in expression was particularly evident for M1 and NP, and justifies largely the choice of codon-optimized sequences for vaccine constructs.

The three transgenes were expressed correctly in both MVA and FPV vectors, yielding proteins of the expected molecular mass. The subcellular localization of the transgenic proteins was analysed by immunofluorescence, which showed three distinct morphological patterns for the three antigens; M2 and NP display characteristic cytoplasmic membrane and nuclear localizations, respectively, while M1 (the influenza protein responsible for the budding of viruses and virus-like particles) displays a vesicular membrane morphology, possibly due to its ability to interact with other M1 molecules to form a continuous shell beneath membranes. The formation of larger vesicles was possibly due to the abnormally high expression of the M1 transgene.

A possible effect of the presence and expression of transgenes on virus growth was investigated by analysing both the burst size and the growth rate of all MVA and FPV vectors and recombinants. No effect could be shown in either case.

The titration of FPV vectors and derivatives on CEF proved to be experimentally complex compared to MVA derivatives, because no clear early CPE is visible and CEF monolayers of cells killed by the virus are not distinguishable macroscopically by crystal violet staining. A modification of the titration method was therefore devised; the original virus-infected CEF titration microcultures supernatants were transferred to BHK-21 microcultures seeded freshly with an inoculum of 500 cells/well. FPV does not grow in BHK-21 cells but does infect and eventually kills them. The virus concentration in the supernatants of infected microcultures is sufficient to sterilize the cell inoculum and prevent monolayer formation.

A real-time PCR method to measure FPV DNA in infected cell cultures was also established, employing primers within the FPV fp9,046 gene, present in the original transfer plasmid pFPwt and used as a control. The method proved to be useful for evaluating growth rate. The relative increase in DNA copies in microcultures infected at low m.o.i. is exponential (e.g., between 18 and 64 h p.i., see Fig. $4 \mathrm{~b}$ ) with the same coefficient of relative increase in virus, as determined by titration using the described above.

FVP derivatives grow significantly slower than MVA derivatives and achieve significantly smaller burst sizes. It would be interesting to evaluate if the longer survival and expression of transgenes in infected cells could be relevant in the vaccination process and support the proposed regimen FPV prime/MVA boost.

\section{Acknowledgements}

This work was supported by Fondazione Cariplo (Iniziativa Vaccini), Ministero della Salute (Unifluvac) and Ministero della Università e Ricerca Scientifica (Progetti CoFin). 


\section{References}

Amara, R.R., Villinger, F., Altman, J.D., Lydy, S.L., O’Neil, S.P., Staprans, S.I., Montefiori, D.C., Xu, Y., Herndon, J.G., Wyatt, L.S., Candido, M.A., Kozyr, N.L., Earl, P.L., Smith, J.M., Ma, H.L., Grimm, B.D., Hulsey, M.L., McClure, H.M., McNicholl, J.M., Moss, B., Robinson, H.L., 2002. Control of a mucosal challenge and prevention of AIDS by a multiprotein DNA/MVA vaccine. Vaccine 20, 1949-1955.

Barouch, D.H., Santra, S., Kuroda, M.J., Schmitz, J.E., Plishka, R., Buckler-White, A., Gaitan, A.E., Zin, R., Nam, J.H., Wyatt, L.S., Lifton, M.A., Nickerson, C.E., Moss, B., Montefiori, D.C., Hirsch, V.M., Letvin, N.L., 2001. Reduction of simian-human immunodeficiency virus $89.6 \mathrm{P}$ viremia in rhesus monkeys by recombinant modified vaccinia virus Ankara vaccination. J. Virol. 75, 5151-5158.

Carroll, M.W., Overwijk, W.W., Chamberlain, R.S., Rosenberg, S.A., Moss, B., Restifo, N.P., 1997. Highly attenuated modified vaccinia virus Ankara (MVA) as an effective recombinant vector: a murine tumor model. Vaccine 15, 387-394.

Carson, C., Antoniou, M., Ruiz-Arguello, M.B., Alcami, A., Christodoulou, V., Messaritakis, I., Blackwell, J.M., Courtenay, O., 2009. A prime/boost DNA/Modified vaccinia virus Ankara vaccine expressing recombinant Leishmania DNA encoding TRYP is safe and immunogenic in outbred dogs, the reservoir of zoonotic visceral leishmaniasis. Vaccine 27, 1080-1086.

Cosma, A., Nagaraj, R., Buhler, S., Hinkula, J., Busch, D.H., Sutter, G., Goebel, F.D., Erfle, V., 2003. Therapeutic vaccination with MVA-HIV-1 nef elicits Nef-specific T-helper cell responses in chronically HIV-1 infected individuals. Vaccine 22, 21-29.

Di Lullo, G., Soprana, E., Panigada, M., Palini, A., Agresti, A., Comunian, C., Milani, A., Capua, I., Erfle, V., Siccardi, A.G., 2010. The combination of marker gene swapping and fluorescence-activated cell sorting improves the efficiency of recombinant modified vaccinia virus Ankara vaccine production for human use. J. Virol. Methods 163, 195-204.

Di Lullo, G., Soprana, E., Panigada, M., Palini, A., Erfle, V., Staib, C., Sutter, G., Siccardi, A., 2009. Marker gene swapping facilitates recombinant Modified Vaccinia Virus Ankara production by host-range selection. J. Virol. Methods 56, 37-43.

Drexler, I., Heller, K., Wahren, B., Erfle, V., Sutter, G., 1998. Highly attenuatedmodified vaccinia virus Ankara replicates in baby hamster kidney cells, a potential host for virus propagation, but not in various human transformed and primary cells. J. Gen. Virol. 79 (Pt 2), 347-352.

Feng, F., Teoh, C.Q., Qiao, Q., Boyle, D., Jilbert, A.R., 2010. The development of persistent duck hepatitis $B$ virus infection can be prevented using antiviral therapy combined with DNA or recombinant fowlpoxvirus vaccines. Vaccine 46 , 7436-7443, Epub 2010 Sep 15.

Grosenbach, D., Barrientos, J., Schlom, J., Hodge, J., 2001. Synergy of vaccine strategies to amplify antigen-specific immune responses and antitumor effects. Cancer Res. 61 (June (11)), 61, 4497-4505.

Hanke, T., Schneider, J., Gilbert, S.C., Hill, A.V., McMichael, A., 1998. DNA multi-CTL epitope vaccines for HIV and Plasmodium falciparum: immunogenicity in mice. Vaccine 16, 426-435.

Hirsch, V.M., Fuerst, T.R., Sutter, G., Carroll, M.W., Yang, L.C., Goldstein, S., Piatak Jr., M., Elkins, W.R., Alvord, W.G., Montefiori, D.C., Moss, B., Lifson, J.D., 1996. Patterns of viral replication correlate with outcome in simian immunodeficiency virus (SIV)-infected macaques: effect of prior immunization with a trivalent SIV 
vaccine in modified vaccinia virus Ankara. J. Virol. 70, 3741-3752.

Hodge, J.W., Poole, D.J., Aarts, W.M., Gomez Yafal, A., Gritz, L., Schlom, J., 2003. Modified vaccinia virus ankara recombinants are as potent as vaccinia recombinants in diversified prime and boost vaccine regimens to elicit therapeutic antitumor responses. Cancer Res. 63, 7942-7949.

Jaoko, W., Nakwagala, F.N., Anzala, O., Manyonyi, G.O., Birungi, J., Nanvubya, A., Bashir, F., Bhatt, K., Ogutu, H., Wakasiaka, S., Matu, L., Waruingi, W., Odada, J., Oyaro, M., Indangasi, J., Ndinya-Achola, J., Konde, C., Mugisha, E., Fast, P., Schmidt, C., Gilmour, J., Tarragona, T., Smith, C., Barin, B., Dally, L., Johnson, B., Muluubya, A., Nielsen, L., Hayes, P., Boaz, M., Hughes, P., Hanke, T., McMichael, A., Bwayo, J., Kaleebu, P., 2008. Safety and immunogenicity of recombinant low-dosage HIV-1 $A$ vaccine candidates vectored by plasmid pTHr DNA or modified vaccinia virus Ankara (MVA) in humans in East Africa. Vaccine 26, 2788-2795.

Kent, S.J., Zhao, A., Best, S.J., Chandler, J.D., Boyle, D.B., Ramshaw, I.A., 1998. Enhanced T-cell immunogenicity and protective efficacy of a human immunodeficiency virus type 1 vaccine regimen consisting of consecutive priming with DNA and boosting with recombinant fowlpox virus. J. Virol. 72, 10180-10188.

Kent, S.J., Zhao, A., Dale, C.J., Land, S., Boyle, D.B., Ramshaw, I.A., 2000. A recombinant avipoxvirus HIV-1 vaccine expressing interferon-gamma is safe and immunogenic in macaques. Vaccine 18, 2250-2256.

Kotsopoulou, E., Kim, V.N., Kingsman, A.J., Kingsman, S.M., Mitrophanous, K.A., 2000. ARev-independent human immunodeficiency virus type 1 (HIV-1)-based vector that exploits a codon-optimised HIV-1 gag-pol gene. J. Virol. 74, 4839-4852.

Malnati, M.S., Scarlatti, G., Gatto, F., Salvatori, F., Cassina, G., Rutigliano, T., Volpi, R., Lusso, P., 2008. A universal real-time PCR assay for the quantitation of group-M HIV-1 proviral load. Nat. Protoc. 3, 1240-1248.

Marshall, J.L., Gulley, J.L., Arlen, P.M., Beetham, P.K., Tsang, K.Y., Slack, R., Hodge, J.W., Doren, S., Grosenbach, D.W., Hwang, J., Fox, E., Odogwu, L., Park, S., Panicali, D., Schlom, J., 2005. Phase I study of sequential vaccinations with fowlpoxCEA(6D)-TRICOM alone and sequentially with vaccinia-CEA(6D)-TRICOM, with and without granulocyte-macrophage colony-stimulating factor, in patients with carcinoembryonic antigen-expressing carcinomas. J. Clin. Oncol. 23, 720-731.

McConkey, S.J., Reece, W.H., Moorthy, V.S., Webster, D., Dunachie, S., Butcher, G., Vuola, J.M., Blanchard, T.J., Gothard, P., Watkins, K., Hannan, C.M., Everaere, S., Brown, K., Kester, K.E., Cummings, J., Williams, J., Heppner, D.G., Pathan, A., Flanagan, K., Arulanantham, N., Roberts, M.T., Roy, M., Smith, G.L., Schneider, J., Peto, T., Sinden, R.E., Gilbert, S.C., Hill, A.V., 2003. Enhanced T-cell immunogenicity of plasmid DNA vaccines boosted by recombinant modified vaccinia virus Ankara in humans. Nat. Med. 9, 729-735.

Pozzi, E., Basavecchia, V., Zanotto, C., Pacchioni, S., De Giuli Morghen, C., Radaelli, A., 2009. Construction and characterization of recombinant fowlpox viruses expressing human papilloma virus E6 and E7 oncoproteins. J. Virol. Methods 158, 184-189.

Radaelli, A., Bonduelle, O., Beggio, P., Mahe, B., Pozzi, E., Elli, V., Paganini, M., Zanotto, C., De Giuli Morghen, C., Combadiere, B., 2007. Prime-boost immunization with DNA, recombinant fowlpox virus and VLP(SHIV) elicit both neutralizing antibodies and IFNgamma-producing T cells against the HIVenvelope protein in mice that control env-bearing tumour cells. Vaccine 25 , 2128-2138. 
Restifo, N.P., Minev, B.R., Taggarse, A.S., McFarland, B.J., Wang, M., Irvine, K.R., 1994. Enhancing the recognition of tumour associated antigens. Folia Biol. (Praha) 40,

74-88.

Schneider, J., Gilbert, S.C., Blanchard, T.J., Hanke, T., Robson, K.J., Hannan, C.M., Becker, M., Sinden, R., Smith, G.L., Hill, A.V., 1998. Enhanced immunogenicity for CD8+ $T$ cell induction and complete protective efficacy of malaria DNA vaccination by boosting with modified vaccinia virus Ankara. Nat. Med. 4, 397-402.

Southern, J.A., Young, D.F., Heaney, F., Baumgärtner, W.K., Randall, R.E., 1991. Identification of an epitope on the $P$ and $V$ proteins of simian virus 5 that distinguishes between two isolates with different biological characteristics. J. Gen. Virol. 72, $1551-1557$.

Steensels, M., Bublot, M., Van Borm, S., De Vriese, J., Lambrecht, B., Richard-Mazet, A., Chanavat-Bizzini, S., Duboeuf, M., Le Gros, F.X., van den Berg, T., 2009. Primeboost vaccination with a fowlpox vector and an inactivated avian influenza vaccine is highly immunogenic in Pekin ducks challenged with AsianH5N1HPAI. Vaccine 27, 646-654.

Sutter, G., Moss, B., 1992. Nonreplicating vaccinia vector efficiently expresses recombinant genes. Proc. Natl. Acad. Sci. U.S.A. 89, 10847-10851.

Sutter, G., Wyatt, L.S., Foley, P.L., Bennink, J.R., Moss, B., 1994. A recombinant vector derived from the host range-restricted and highly attenuated MVA strain of vaccinia virus stimulates protective immunity in mice to influenza virus. Vaccine $12,1032-1040$.

Taylor, J., Weinberg, R., Languet, B., Desmettre, P., Paoletti, E., 1988. Recombinant fowlpox virus inducing protective immunity in non-avian species. Vaccine 6 , 497-503.

Tsang, K.Y., Palena, C., Yokokawa, J., Arlen, P.M., Gulley, J.L., Mazzara, G.P., Gritz, L., Yafal, A.G., Ogueta, S., Greenhalgh, P., Manson, K., Panicali, D., Schlom, J., 2005. Analyses of recombinant vaccinia and fowlpox vaccine vectors expressing transgenes for twohumantumor antigens and threehumancostimulatory molecules. Clin. Cancer Res. 11, 1597-1607.

Wang, M., Bronte, V., Chen, P.W., Gritz, L., Panicali, D., Rosenberg, S.A., Restifo, N.P., 1995. Active immunotherapy of cancer with a nonreplicating recombinant fowlpox virus encoding a model tumor-associated antigen. J. Immunol. $154,4685-4692$.

Webster, D.P., Dunachie, S., McConkey, S., Poulton, I., Moore, A.C., Walther, M., Laidlaw, S.M., Peto, T., Skinner, M.A., Gilbert, S.C., Hill, A.V., 2006. Safety of recombinant fowlpox strain FP9 and modified vaccinia virus Ankara vaccines against liver-stage $P$. falciparum malaria in non-immune volunteers. Vaccine 24 , 3026-3034.

Weidinger, G., OhImann, M., Schlereth, B., Sutter, G., Niewiesk, S., 2001. Vaccination with recombinant modified vaccinia virus Ankara protects against measles virus infection in the mouse and cotton rat model. Vaccine 19, 2764-2768.

Wyatt, L.S., Shors, S.T., Murphy, B.R., Moss, B., 1996. Development of a replicationdeficient recombinant vaccinia virus vaccine effective against parainfluenza virus 3 infection in an animal model. Vaccine 14, 1451-1458. 


\section{Figures}

Figure 1. Constructionsof the acceptor virus FPD-Red. (a) Schematic diagram of genetic constructs. (b) Sorting and cloning of FPD-Red. A cell lysate from infection/transfection of CEF with FPwt and pFP-Dual-Red Transfer Plasmid was diluted 1/50 and used to infect a fresh CEF culture in the presence of $1 \mathrm{mM}$ cytochalasin D. Flow cytometry analysis at $18 \mathrm{~h} \mathrm{p.i.} \mathrm{showed} \mathrm{a} \mathrm{fraction} \mathrm{(ca.} \mathrm{1 \% )} \mathrm{of}$ red cells (Sort Window). (c) Whole-plate fluoroimaging of microtiter terminal dilution of sorted red cells infected by recombinant virus. (d) Fluorescent microscopy of red foci in CEF infected monolayers.

a
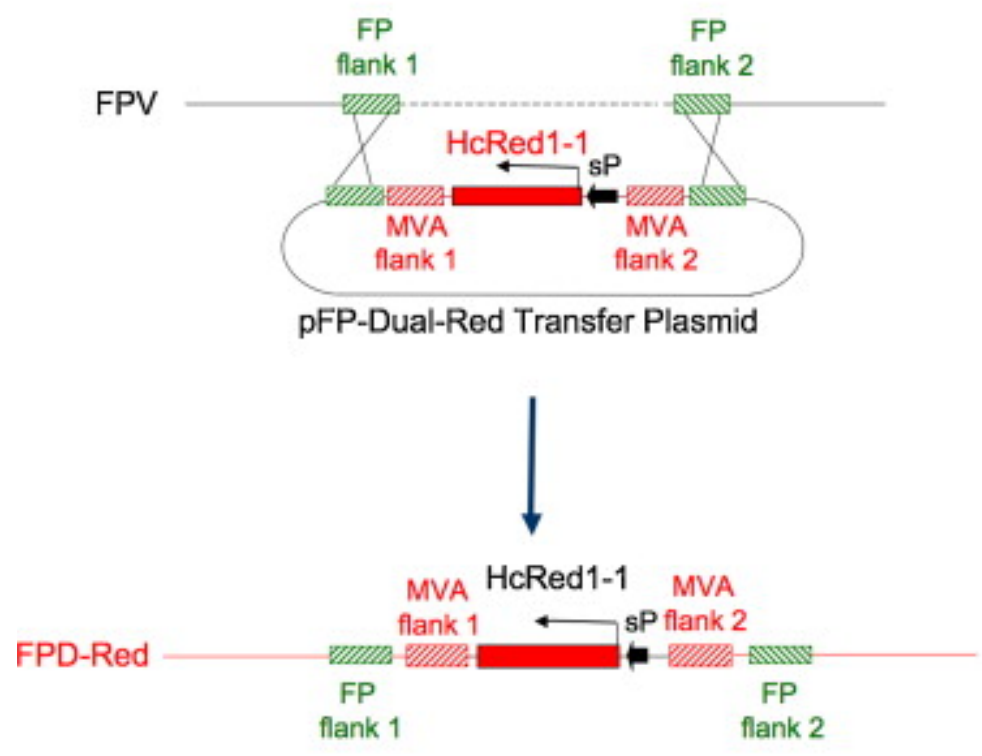

C

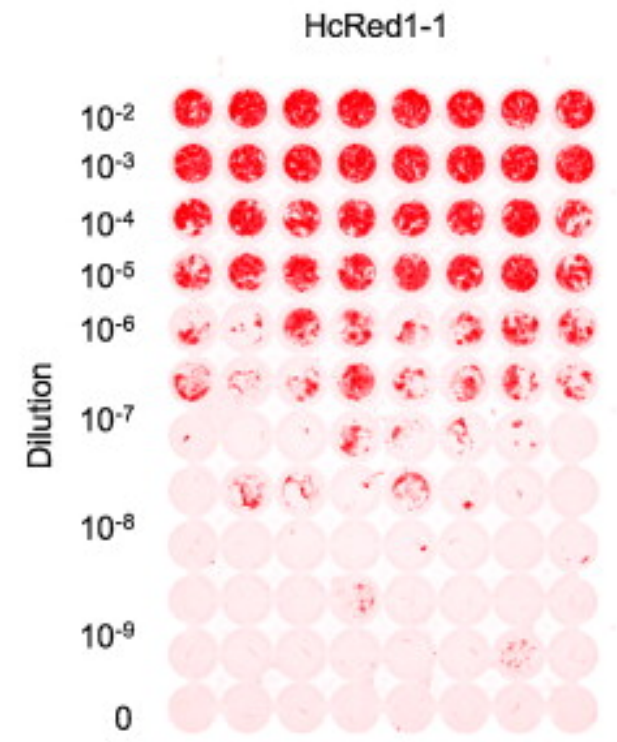

b

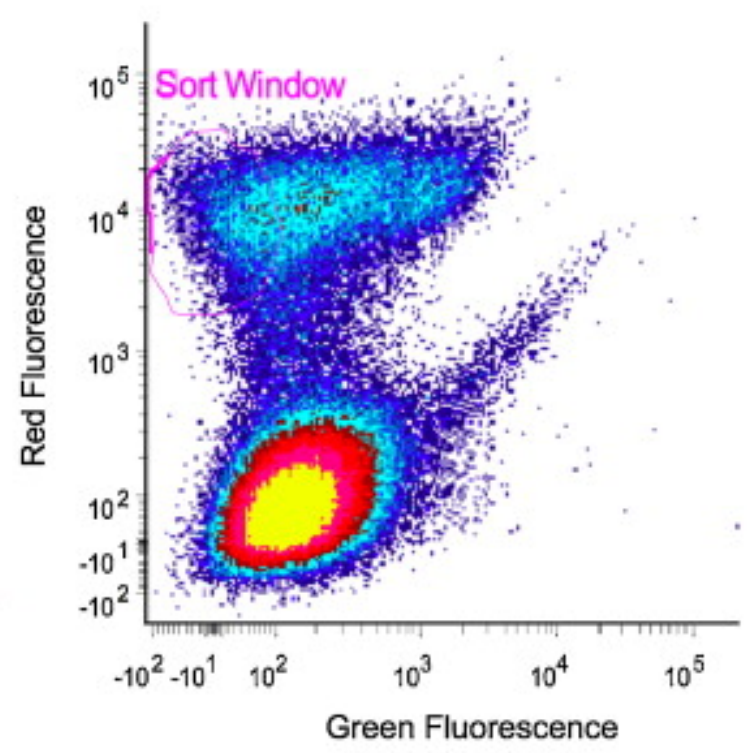

d
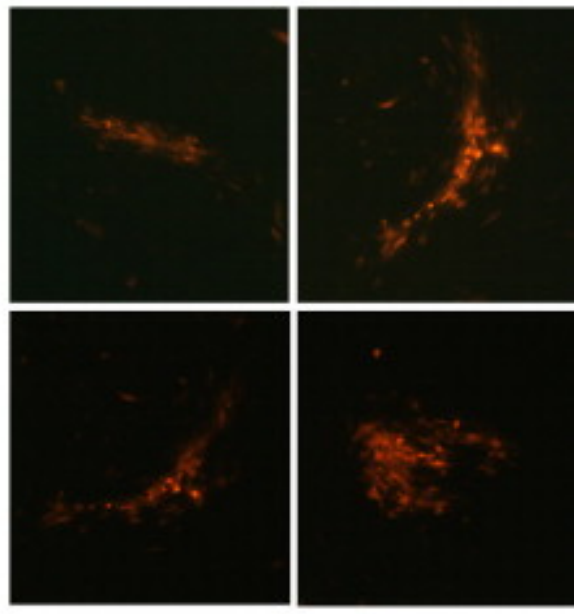
Figure 2. Construction of recombinant MVA and FPD. (a) V5 Western blot analysis of wild-type (wt) and human codon-optimized (co) M1, M2 and NP genes in PTH plasmids after transfection in 293T cells. (b) Schematic diagram of genetic constructs. Maps of viral vector FPD-Red and Tranfer Plasmid Green. (c) Sorting and cloning of recombinant viruses. Cell lysates from infection/transfection of CEF with MVA-Red or FPD-Red and Transgene-carrying Transfer Pasmid Green DNA were diluted 1/50 and used to infect fresh CEF cultures in the presence of $1 \mathrm{mM}$ cytochalasin D. Flow cytometry analysis at $18 \mathrm{~h}$ p.i. showed a small fraction $(<0.01 \%)$ of green cells (Sort Window). (d) Terminal dilution of sorted green cells analysed by whole-plate fluoroimaging of the original microcultures and by crystal violet staining of a replica plate. Most of the clones are green, but two (microtiter cultures $\mathrm{D} 10$ and $\mathrm{E} 9$, circled by dotted lines) are colorless.

a

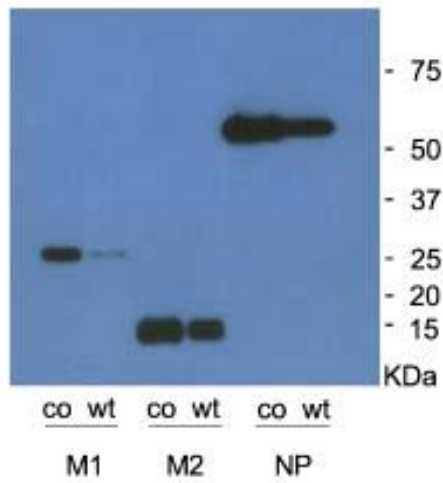

C

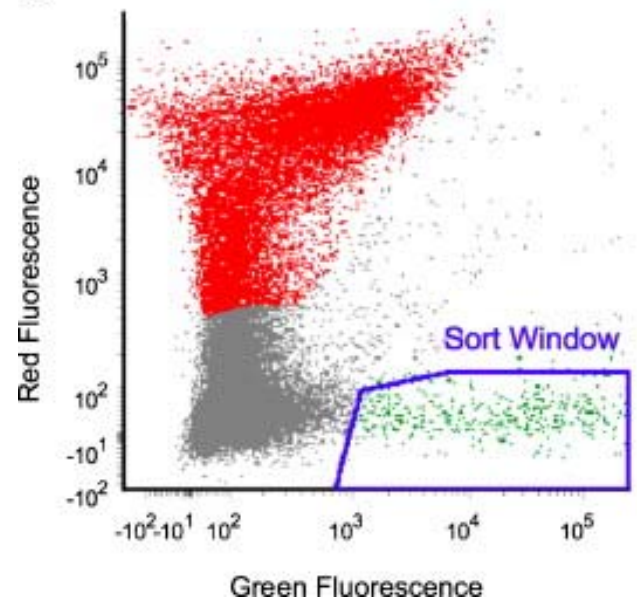

b

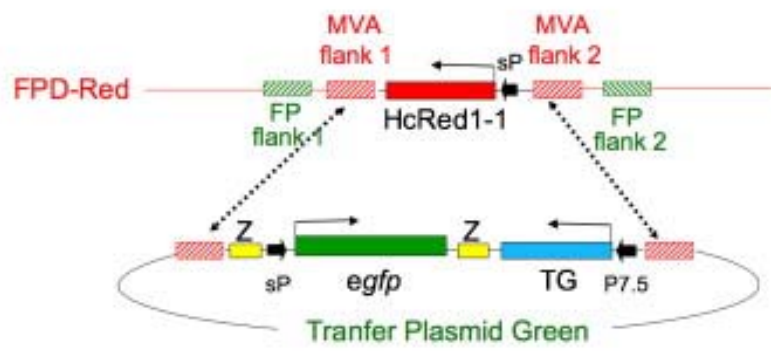

d

EGFP

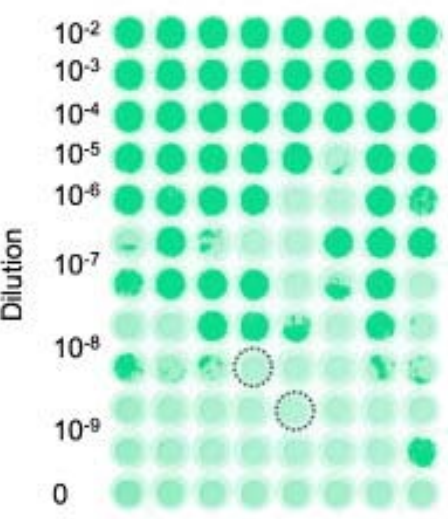

CV

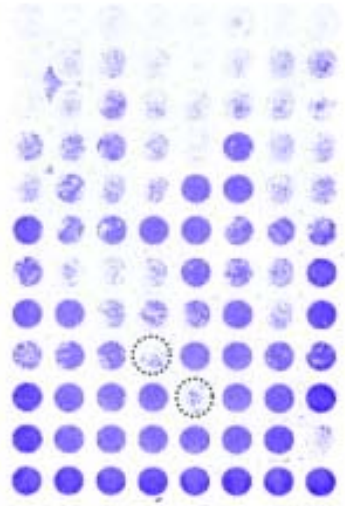


Figure 3. Characterization of recombinant viruses expressing H5N1 proteins. (a) Transgene (M1, M2, $\mathrm{NP}$ ) expression by MVA recombinants in infected CEF, monitored by V5 immunofluorescence (red), Hoechst nuclear staining (blue), merging and phase contrast. (b) V5 Western blot analysis of rMVA and rFPV derivatives. The arrows indicate the expected molecular masses. (c) V5 immunoperoxidase staining of infected foci in CEF monolayers.

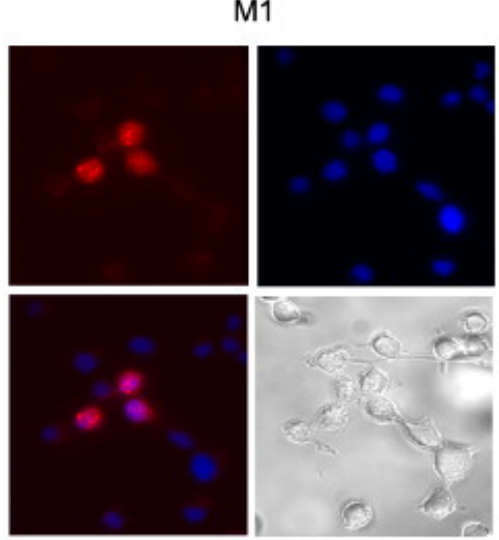

M2
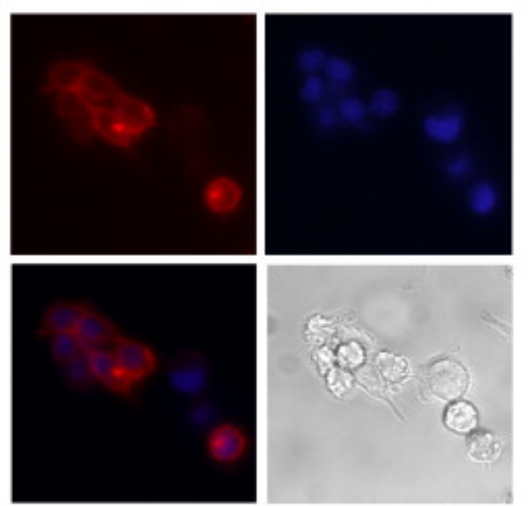

NP
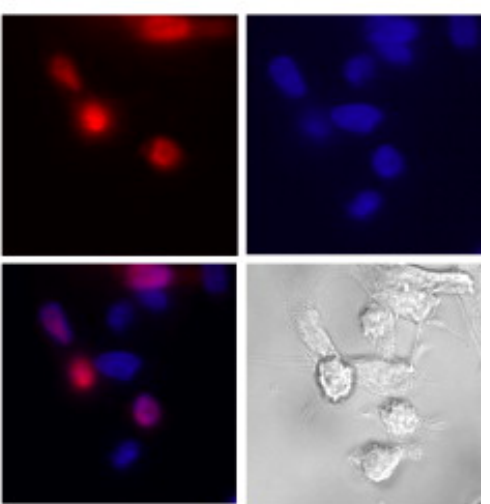

b

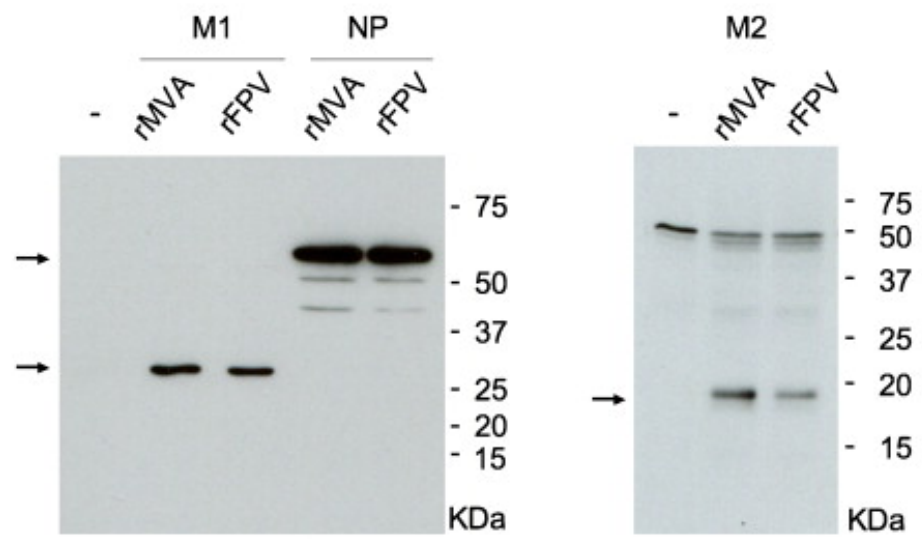

WB $\alpha$ V5
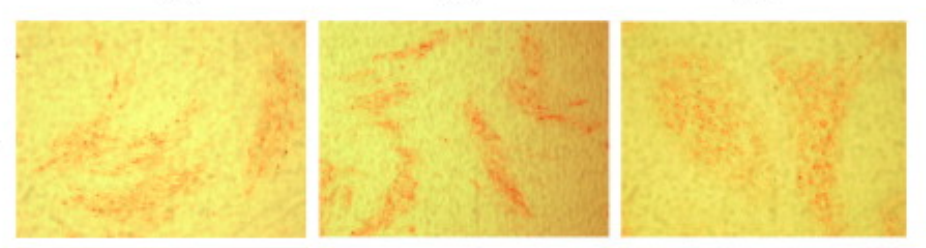

D
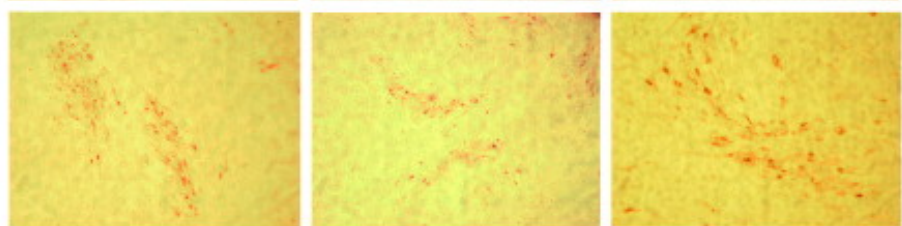
Figure 4. Growth analysis of recombinant virus. (a) Growth rate of MVA derivatives analysed by virus titration. The exponential growth curves of all the derivatives could be described with a single equation (coefficient $=0.226 ; R^{2}=0.96$ ). (b) The growth rate of FPV/FPD derivatives analysed by virus titration (-TIT) and by real-time PCR (-RT). The exponential growth curves of all the derivatives, by both methods, could be described with a single equation (coefficient $=0.116 ; R^{2}=0.97$ ). $D t$ is the doubling time calculated from the curves.
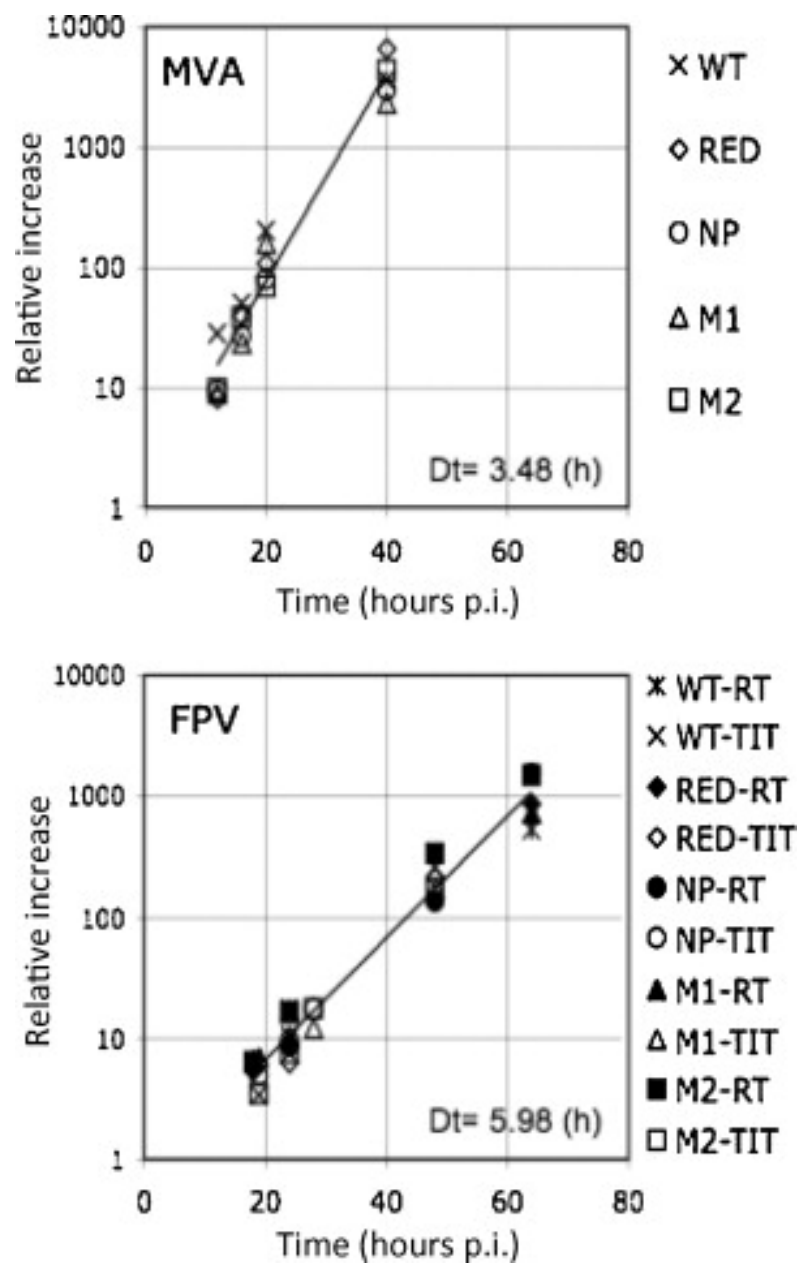